\title{
La culture matérielle, la numérisation et le problème de l'artefact
}

Material Culture, Digitization and the Problem of the Artefact.

\section{Victor Buchli}

Traducteur : Manuel Benguigui

\section{OpenEdition Journals}

Édition électronique

URL : https://journals.openedition.org/tc/4743

DOI : $10.4000 /$ tc. 4743

ISSN : $1952-420 X$

Éditeur

Éditions de l'EHESS

\section{Édition imprimée}

Date de publication : 1 décembre 2009

Pagination : 212-231

ISBN : 978-2-7351-1301-9

ISSN : 0248-6016

\section{Référence électronique}

Victor Buchli, «La culture matérielle, la numérisation et le problème de l'artefact », Techniques \& Culture [En ligne], 52-53 | 2009, mis en ligne le 01 août 2012, consulté le 29 septembre 2022. URL : http:// journals.openedition.org/tc/4743; DOI : https://doi.org/10.4000/tc.4743

Ce document a été généré automatiquement le 29 septembre 2022.

Tous droits réservés 


\section{La culture matérielle, la} numérisation et le problème de l'artefact ${ }^{1}$

Material Culture, Digitization and the Problem of the Artefact.

\section{Victor Buchli}

Traduction : Manuel Benguigui

\section{NOTE DE L'ÉDITEUR}

Victor Buchli analyse dans cet article comment les nouveaux procédés de fabrication questionnent nos convictions et perceptions du monde et du réel. 


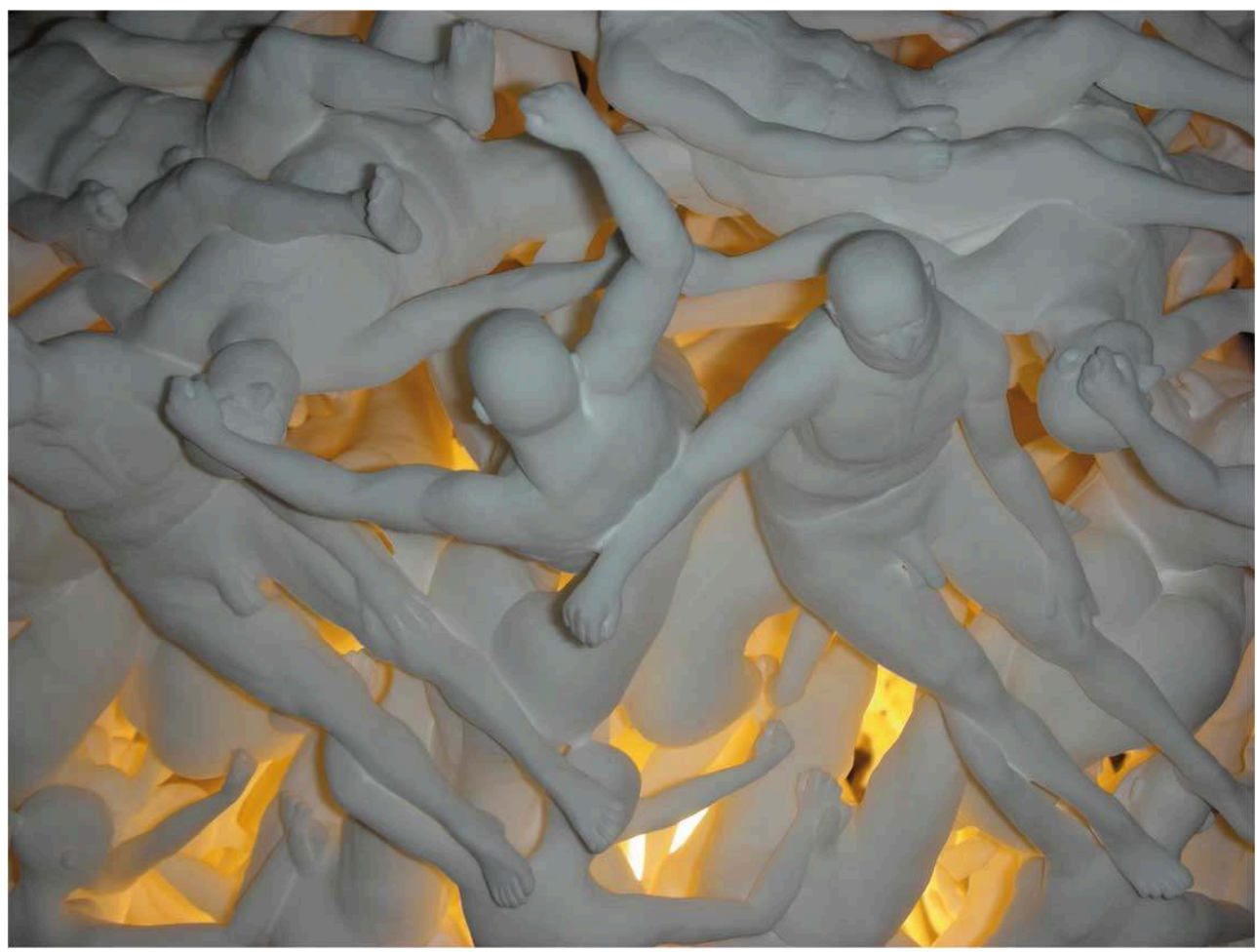

¿ Materialise

Les techniques de Fabrication rapide et de Prototypage rapide ("Rapid Manufacturing ", en anglais) sont au cœur d'une technologie "immatérielle » fondée sur des poudres informes et sur un code digital éphémère. En fait, le seul élément véritablement stable de cette technologie est son code digital «immatériel»: paradoxalement, sa production par itération est beaucoup plus instable. En raison des défis radicaux qu'elle pose à notre conception de la fabrication et de la matérialité (Hopkinson \& al. 2006), les observateurs de l'industrie ont présenté son apparition comme une seconde révolution industrielle. Émergeant du domaine du Prototypage rapide, qui se développe à grande vitesse, cette nouvelle technologie permet de produire des objets tridimensionnels de n'importe quelle forme imaginable, chose impossible jusque-là, qui offre, selon les ingénieurs, une " liberté géométrique » totale (Hopkinson \& al. 2006). Pour résumer la chose, on commence par concevoir un objet en trois dimensions sur un ordinateur (en CAD [Computer Aided Drafting Software]), puis l'information digitale (transformée en un fichier stl [Standard Triangulation Language]) est utilisée pour fabriquer l'objet par addition d'éléments, contrairement aux méthodes soustractives conventionnelles. Ce procédé est possible grâce à un support hautement fluide, comme des polymères en poudre ou certains métaux (parmi d'autres composants possibles) et à une technique par micro-couches (comme le frittage au laser ou la stéréolithographie). Le résultat de ces opérations est un artefact tridimensionnel. La taille des imprimantes tridimensionnelles employées pour parvenir à ce résultat peut aller de celle d'une petite photocopieuse (sorte d'« usine " personnelle indépendante, telle la Perfactory ${ }^{\circledast}$ ) à des machines assez grosses pour imprimer une image tridimensionnelle de la taille d'un homme, ou encore bien plus grosses, sur lesquelles on peut imprimer des éléments architecturaux et aéronautiques. L'image CAD peut être créée puis transmise numériquement n'importe où dans le monde, pour être réalisée de façon virtuellement instantanée selon l'échelle et le support indiqués. Aucun matériau solide n'est manipulé 
- il ne s'agit que d'un code digital binaire, d'éléments en poudre et de liquides. Ses usages sont extrêmement variés, puisque cette technologie permet de produire aussi bien des prothèses médicales que des composants automobiles, des abat-jour, des pièces de remplacement pour les missions spatiales, des ailes d'avion ou encore des coupes à fruits.

1 Décrite comme une "technologie perturbatrice " par de nombreux observateurs et développeurs qui considèrent qu'elle bouleverse les conceptions et les pratiques de fabrication traditionnelles (Hopkinson \& al. 2006), la Fabrication rapide pose une série de problèmes pour les conceptions conventionnelles euroaméricaines de la matérialité d'aujourd'hui. La Fabrication rapide vient bouleverser nos conceptions dualistes occidentales telles que la séparation ontologique entre matière vivante et matière morte, pensée et chose, nature et culture, sujet et objet, créativité et authenticité. La possibilité d'imprimer des objets en trois dimensions permet d'éviter presque entièrement les contraintes conventionnelles de matière et de fabrication (Hopkinson $\&$ al. 2006). Grâce à cette technologie, il est possible de construire des objets solides qui peuvent être composés de matériaux hybrides (céramique, polymère et métal, par exemple) tous fusionnés au niveau micro. Implants dentaires, prothèses auditives et médicales -constituent les principales applications sur le plan commercial. Les ossements humains, les dents, etc., peuvent être scannés puis imprimés en trois dimensions, dans des matériaux variés, pour créer des prothèses adaptées aux mensurations précises de n'importe quel individu. Des machines qui permettent une impression tridimensionnelle de tissu humain à base de cellules vivantes prêt à l'implantation (modèle 3D Bioplotter de la marque Envisiontec, par exemple) sont aujourd'hui disponibles dans le commerce.

2 La Fabrication rapide remet donc en question un grand nombre d'idées dominantes concernant les artefacts et leur signification sociale, ainsi que nos conceptions de la présence. Je propose dans cet article de développer une conception alternative de la présence ou de la co-présence que je nommerai "propinquité ", sur la base du mot anglais ("propinquity»). Ce mot désigne une relation d'analogie, de proximité temporelle et de proximité spatiale, plutôt que de co-présence visuelle et physique, comme ce serait le cas si nous parlions en termes empiriques familiers. En outre, cette technologie rend problématique notre conception des artefacts, autrefois envisagés comme étant les reflets passifs de nos intentions et plus récemment selon leur solidité et leur capacité à résister à nos actions en raison de leur matérialité multi-couches - ce que Keane appelle «bundling 》 (Keane 2005). Cette résistance a été considérée comme le signe d'un certain agent indépendant qui aurait la forme d'une donnée irréductible façonnant notre univers social et notre univers matériel. Les technologies de Fabrication rapide remettent en question ces conceptions traditionnelles de résistance matérielle dans le cadre de l'anthropologie et des sciences sociales. Au sein de cette technologie émergente, il semble que les objets ne montrent plus aucune résistance. En fait, il devient possible, contrairement aux restrictions imposées par la fabrication traditionnelle, de faire coïncider presque exactement l'idée de départ et le résultat. Les contraintes matérielles rencontrées dans la fabrication machinique contemporaine sont négligeables, voire inexistantes.

3 La Fabrication rapide remet en question les grands édifices sociaux de la première révolution industrielle, avec les hiérarchies et les inégalités sociales qui leur sont associées et qui ont été critiquées de façon si incisive par Marx et les spécialistes des 
sciences sociales qui se sont succédé depuis. La Fabrication rapide remet également en question les structures sociales classiques associées à la production: le créateur et le producteur, le travailleur et le capitaliste par exemple, physiquement séparés l'un de l'autre.

4 Sous cet éclairage, des questions de proximité - plutôt que celle plus conventionnelle de "présence» en rapport avec les problèmes de travail et de migration, de flux mondiaux d'investissement et de capital - surgissent dès lors que les artefacts peuvent être achetés en ligne, instantanément personnalisés puis fabriqués n'importe où dans le monde. Le récent -projet, lancé par la Rabih Hage Gallery de Londres en collaboration avec la compagnie allemande EOS (développeur et producteur dans le domaine de la 3D), de création par le procédé de Fabrication rapide d'une grande structure architecturale, illustre bien le phénomène.

5 Comme le suggère clairement le dossier de presse de la galerie en mars 2008

«[...] les données (dessins) CAD peuvent être envoyées par courrier électronique. Ces données peuvent être utilisées pour fabriquer la structure sur une machine électronique de Fabrication rapide n'importe où dans le monde, évitant ainsi tous frais de livraison et toutes taxes $»$.

6 Toutes les idées d'espace, de géographie, de temps, d'État-nation, de régime d'imposition et de marché du travail, et avec elles les accords syndicaux, sont dépassées par un simple clic de souris. La "vicieuse bifurcation " (Whitehead 2000) qui a formé nos conceptions du monde empirique et a alimenté les dualismes structurant la vie sociale, ainsi que les « objets » et les « sujets » qui en découlent, sont quasiment réunis, et en même temps dissous, dès lors que représentation numérique et chose physique deviennent difficiles à différencier dans le temps et l'espace. Les dualismes au fondement de nos systèmes de production industriels s'effacent et c'est pour cela que l'on pourrait peut-être même considérer les processus de Fabrication rapide comme des indices d'une « seconde révolution industrielle». 


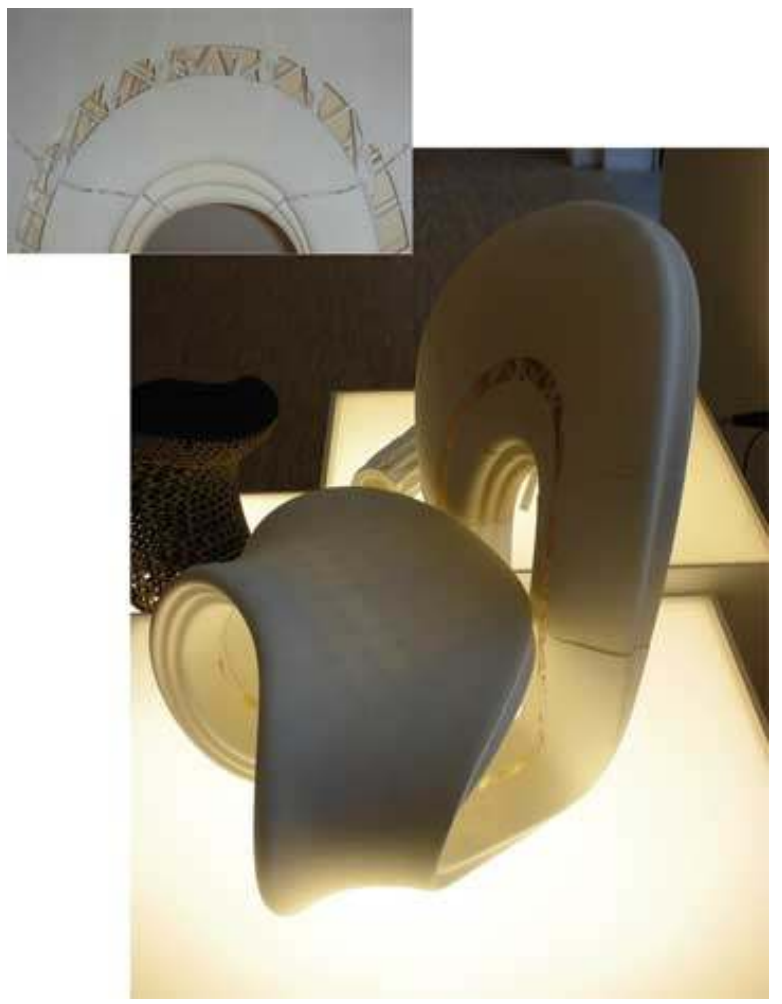

(B) Materialise

Chaise créée par Assa Aschuach, fabriquée par .MGX pour Materialise.

7 Les phénomènes que nous venons de décrire sont très proches de ce que nous allons voir pour l'icône chrétienne et son prototype. La propinquité, en termes de co-présence physique et visuelle, devient alors floue, puisque l'on considère que l'artefact existe dans un contexte spatial et temporel qui se trouve en dehors des conceptions familières de la présence empirique.

Ceci conduit à un autre problème : celui des termes nouveaux par lesquels les individus et les choses sont reconfigurés et assemblés. La Fabrication rapide, qui émerge au sein des bouleversements sociaux générés par la mondialisation néolibérale et la numérisation, nous force à repenser nos anciennes conceptions des relations sociales qui sont ancrées dans des notions traditionnelles de production et d'échange, avec leurs dualismes productifs afférents. Par exemple, le problème classique de l'aliénation du travailleur et de la désagrégation des liens sociaux est potentiellement exacerbé par l'individuation extrême suggérée par le système Perfactory ${ }^{\circledast}$ de la marque Envisiontec. La ville industrielle classique du $\mathrm{xIX}^{\mathrm{e}}$ siècle et ses institutions de travail et de vie sociale, ses vastes paysages, ses réseaux de distribution et ses infrastructures matérielles qui peuvent produire une " chose » donnée, sont supplantés par une " usine personnelle » hautement individualisée et placée dans un bureau. Les flux normaux de la mondialisation sont dès lors bouleversés, dans le sens où il devient possible de créer des objets dès que l'on en a besoin, à n'importe quel moment, au lieu de les produire à un endroit donné avec des outils et des matériaux traditionnels avant de les transporter (par voie terrestre, aérienne ou maritime) jusqu'au point d'usage.

9 À l'instar de nos conceptions conventionnelles de la "vie ", les notions de "réel ", de «virtuel » et d'« authentique » deviennent instables et sont remises en question dès 
lors qu'on peut envisager l'« impression » de tissu humain. Il en va de même pour les notions de temps associées à l'objet manufacturé, fondé sur un prototype passé et sur l'expérience accumulée, les contingences prévues, ou induites. Les notions anthropologiques traditionnelles de «consommation", d'«appropriation ", d'«authenticité " et d'«aliénation», utilisées dans les sciences sociales, sont entièrement reconfigurées alors même que la tendance croissante à la "personnalisation de masse" dans la fabrication et ses hiérarchies sociales et politiques afférentes sont fusionnées avec la Fabrication rapide. C'est le dilemme auquel doivent faire face les fabricants lorsqu'ils prennent en compte le fait qu'une marchandise de marque est personnalisée par un client particulier à un tel degré que la marchandise peut finir par ne plus être identifiée comme étant de sa marque d'origine. De plus, le temps prend une dimension sociale nouvelle et inexplorée. Comme l'on pourrait s'y attendre dans une reconfiguration d'une telle radicalité, l'ancienne perception de la temporalité n'est plus en vigueur. Les modes linéaires utilisés auparavant pour envisager des scénarios futurs et généralement fondés sur des grilles cumulatives d'expériences antérieures sont remplacés par un futur ouvert et indécis. Les besoins non anticipés peuvent être satisfaits à des moments non anticipés. Mais dans ce contexte, toutes les contingences deviendraient-elles maitrisables?

Comme nous allons le voir, de la même façon que l'émergence du prototype chrétien a créé un cadre radicalement nouveau dans lequel il était possible d'actualiser le divin de façon universelle au sein d'un cadre temporel et géographique distinct, la Fabrication rapide produit un cadre nouveau pour la nature de l'artefact et sa temporalité. Si le seul noyau stable est au final le code numérique et si ses itérations lors de la réalisation en trois dimensions sont techniquement infinies et illimitées, quel est alors le statut de l'artefact (son existence réelle?) dans l'espace et dans le temps? Que signifie également la 3D ? Comment l'artefact peut-il être défini et décrit si la seule entité stable est le code - en tant que fichier stl ou en tant qu'accrétion donnée sous une forme physique, en termes de co-présence physique et visuelle ? Et quels types d'engagements sociaux et de collectivités peuvent émerger de tels processus? Quel peut alors être le statut de l'empirique ? Les questions concernant le simulacre et l'aura de Benjamin ou celle de l'«artefact distribué » ("ditributed artefact») de Gell voient leur importance renouvelée au sein de cette technologie perturbatrice. Cette situation est très proche de celle du prototype chrétien qui, au début de l'ère chrétienne, avait révolutionné la conception de la présence en termes de " proximité » (ou de propinquité).

Il semblerait que sous de nombreux aspects le registre matériel annoncé par des technologies nouvelles, telles que la Fabrication rapide, constitue l'expression parfaite de la matérialité du néolibéralisme, avec ses capacités à produire des formes extrêmes d'individuation. Mais comme de nombreux observateurs (Povinelli 2001; Ong 1967; Sassen 2007; Rose 1998) l'ont remarqué, de telles technologies néolibérales de la personne sont profondément infléchies au niveau local. De même, le développement de la Fabrication rapide, aussi fluide et universalisante puisse-t-elle sembler, a lieu au sein de cadres spécifiques et revêt des dimensions nouvelles et inattendues précisément à cause de son registre matériel spécifique et de ses inflexions locales. Comme nous l'avons déjà vu par le passé avec d'autres innovations, tout porte à penser qu'il en ira de même avec ce nouveau registre de l'immatérialité apparente. 
Hiden .MGX

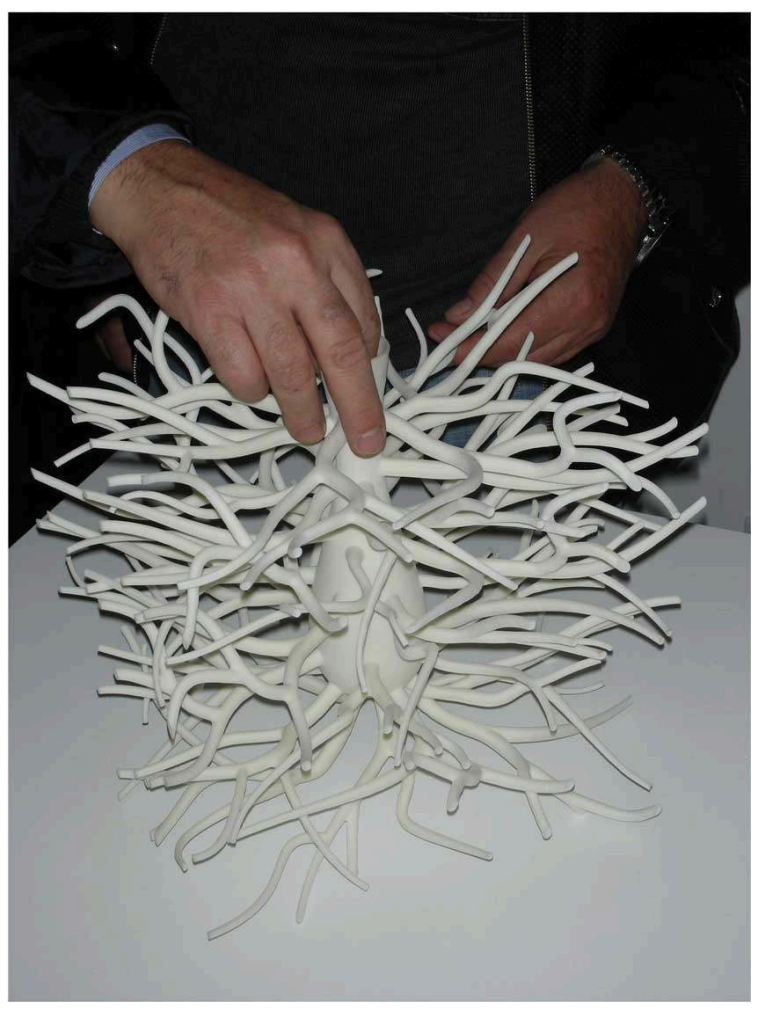

(B) Materialise

Vase créé par Dan Yeffet, fabriqué par .MGX. pour Materialise.

Il y a bien sûr une certaine résistance matérielle inattendue dans cette forme d'apparente "immatérialité » : la qualité irrégulière des surfaces, si utile dans certaines applications médicales pour son imitation des surfaces naturellement rugueuses des os, résulte directement de la forme de l'information, c'est-à-dire la triangulation des fichiers stl et sa découpe fine, à l'horizontal, le long de l'axe des z, vertical. Cependant, alors que les choses sont disposées en couches, cet axe suggère une dimension matérielle et une résistance façonnant l'objet d'une manière spécifique et unique à cette technologie - la création d'une nouvelle forme de résistance différente de nos conceptions conventionnelles. Les principaux domaines du dévelop-pement futur de cette technologie ne sont pas du côté de la fabrication - ces questions étant plus ou moins résolues - mais du côté du développement de la programmation de logiciels (Hopkinson \& al. 2006) qui formatent les données, puis façonnent l'objet par apports réguliers de matière, comme pour les contours irréguliers produits sur l'axe vertical. Les contraintes de fabrication et la rétroactivité des matériaux ne sont plus directement liées aux actions de mise en forme mais à la nature même des composants utilisés, qu'il s'agisse de poudre ou de liquide, et des moyens par lesquels l'information numérisée est créée et appliquée. Pensée et chose ne sont plus dans une relation dichotomique l'une vis-à-vis de l'autre, mais sont en fait mutuellement constitutives l'une de l'autre. L'insistance traditionnelle sur l'immatérialité de la pensée et de l'information et leur mise en opposition sont remises en cause lorsque le signe et le signifiant, la pensée « immatérielle » et la chose «matérielle », deviennent difficiles à distinguer et à démêler. Cette technologie représente une "perturbation » qui va bien au-delà d'une simple remise en question des processus de fabrication classique. Comme 
nous le savons, l'impact social de la première révolution industrielle s'est avéré bien plus important que la seule production de marchandises. Un exemple tiré de l'industrie aérospatiale montre comment la localisation extrême de la production, où la conception et la fabrication peuvent se dérouler en un même temps, réduit de façon radicale la valeur du travail tant en termes de transport, de fabrication et de contrôle de qualité. Ces nouveaux processus de fabrication bouleversent profondément les relations de travail traditionnelles (voir Hopkinson \& al. 2006). Si ces impacts ne se font ressentir qu'aujourd'hui, ils promettent d'être plus nets et complexes à l'avenir, puisque les intérêts croissants de l'industrie pour la conception de pointe, la production aérienne, la production automobile, les prothèses médicales et dentaires, etc., continuent d'exploiter et de faire avancer cette technologie. Dans le même temps, les idées d'authenticité, de fiabilité, de paternité et de diffusion mondialisée sont remises en question par le développement de cette technologie (voir Hopkinson \& al. 2006).

Damned .MGX

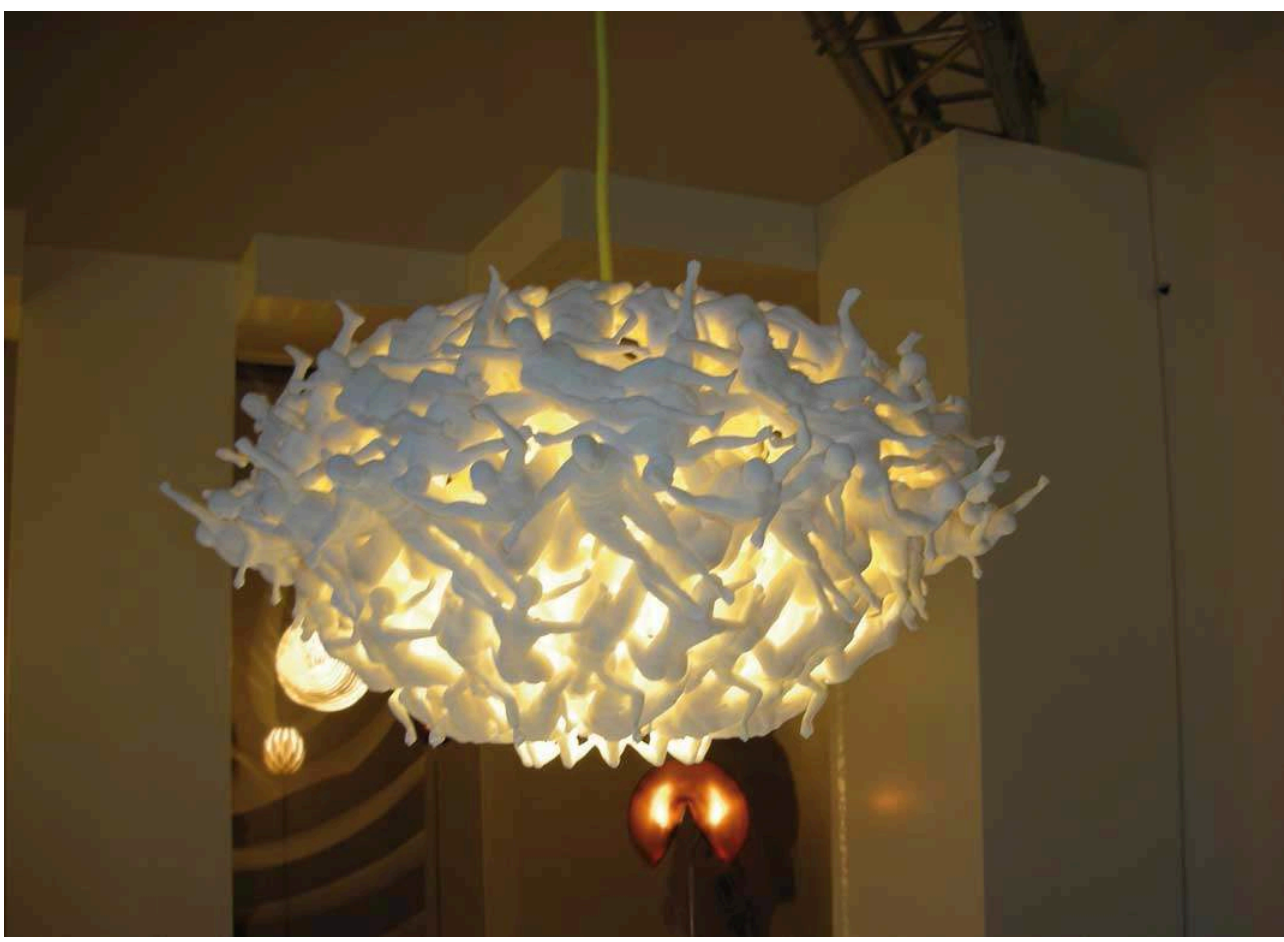

( ) Materialise

Lustre créé par Luc Merx, fabriqué par.MGX pour Materialise.

En outre, les conditions actuelles du marché et les traditions de fabrication structurent les façons dont la technologie est conçue, émerge puis se diffuse au sein de l'industrie et des consommateurs. Les spécialistes en marketing doivent trouver leur place dans ces nouveaux contextes. Dans la Fabrication rapide, un registre différent de matériau apparaît. Il a des caractéristiques spécifiques de résistances, qui ne sont pas anticipées par les ingénieurs et les concepteurs mais constituent une conséquence directe des matériaux utilisés, de la forme des informations et des imprimantes et machines qui exécutent ces ordres. Notre remarque initiale sur les poudres de polymères et la relative fragilité des matériaux proches des os et du verre a invité à penser à une trajectoire spécifique d'émergence dans le secteur de pointe ou haut de gamme. C'est 
au sein de ces cadres que la Fabrication rapide apparait dans les installations légères de conception haut de gamme, avec pour référence le prix de verres artisanaux aussi délicats et translucides que ceux de Murano, par exemple, ou, comme dans la texture de prothèses médicales résultant de l'axe vertical de la modélisation en CAD qui produit de petits rebords irréguliers analogues à ceux des os humains. Les limites instaurées par les syndicats, les échelles économiques de production et la propriété intellectuelle permettront à cette technologie potentiellement universelle de satisfaire les conditions d'émergence d'intérêts locaux. On pourra alors s'attendre à ce qu'elles se réorganisent selon de nouvelles configurations.

\section{Le prototype chrétien}

Comme mentionné plus haut, il existe des similarités importantes entre cette technologie récente du prototype qu'est la Fabrication rapide et les technologies plus anciennes $\mathrm{du}$ prototype chrétien. Ces similarités se -révèlent particulièrement pertinentes dans le cadre de la question historique des icônes et des idoles. Lorsqu'une idole est détruite, la divinité qui lui est consubstantielle est elle aussi détruite. En revanche, les icônes fonctionnent de manière différente: elles renvoient à un prototype dont elles diffusent la présence. Dans le registre de la co-présence visuelle et physique, le prototype est absent : il n'est tout simplement pas là. C'est pourquoi on peut détruire une icône sans affecter le prototype. Lorsqu'une icône du Christ ou une icône de l'empereur byzantin est détruite, ni le Christ ni l'empereur n'en sont généralement pas affectés. L'artefact physique est simplement un intermédiaire par lequel la proximité est atteinte de façon haptique (par le toucher) par le biais de l'icône.

Afin d'illustrer le fonctionnement de ce phénomène, relisons, dans sa version rapportée par l'historien de l'art byzantin, Barber, l'homélie prononcée par le patriarche Photios en 867 à l'occasion de l'inauguration de la mosaïque absidale de l'intronisation de Theotokos dans la basilique Sainte-Sophie de Constantinople. La visualité haptique de l'icône joue le rôle d'intermédiaire par lequel le spectateur, l'icône et le prototype divin interagissent pour présentifier le divin :

«[...] Plus grande est la puissance du regard. Car il est certain que, dès lors que la chose vue est touchée et caressée par le déversement et l'émanation des rayons optiques, la forme de la chose vue est envoyée dans l'esprit, puis transmise à la mémoire pour l'accumulation d'un savoir infaillible » (Patriarche Photios 867, in Barber $2002: 136$ ).

La notion classique de "vision haptique» envisage la vision comme une forme supérieure du toucher : les particules de lumière émanent de ce qui est vu et touchent effectivement celui qui regarde. Dans le même ordre d'idées, une autre métaphore, dérivée de la tradition aristotélicienne, utilisait l'image du sceau - par lequel l'image de ce qui est vu est imprimée, tel un sceau, sur le spectateur - pour traduire l'impression de la vision sur l'âme, " de la même manière que l'on applique un cachet à l'aide d'une bague» (Frank 2000: 125). Ces deux métaphores expriment une conception selon laquelle la vue est une forme de toucher physique, avec toutes les implications en termes de diffusion ou même de " contagion » que cela peut comporter.

L'élément commun de ces diverses notions antiques est la question du toucher, que son origine soit l'œil (avec projection des rayons vers l'extérieur) ou que l'œil en soit la cible (les particules ou le «cachet » s'imprimant sur lui). Si l'on suit Frank, selon qui 
«le plus dangereux était le pouvoir de la vision de connecter le spectateur à l'objet d'une façon si intime que l'adhésion pouvait abîmer l'âme au-delà de toute restauration » (Frank 2000 : 131), on peut dire qu'il y a une puissance et un danger tout aussi grand dans la qualité haptique de la vision. Comme elle le remarque par ailleurs en citant Nemesius, évêque d'Emèse, la vue et le toucher englobent tous deux les propriétés clés « du contact, de la participation et de l'initiative » (Ibid:132). Cette idée rejoint ce qu'elle appelle la "piété tactile ", centrée sur la vue et représentant une convergence des sens alors que, pour les sensibilités modernes, les sens sont isolés, d'où un cadre physique et épistémique totalement différent. La vision permettait un contact tactile direct avec le divin lors de ces rencontres, "c'était une forme de contact physique entre le spectateur et l'objet » (Frank 2000 : 133).

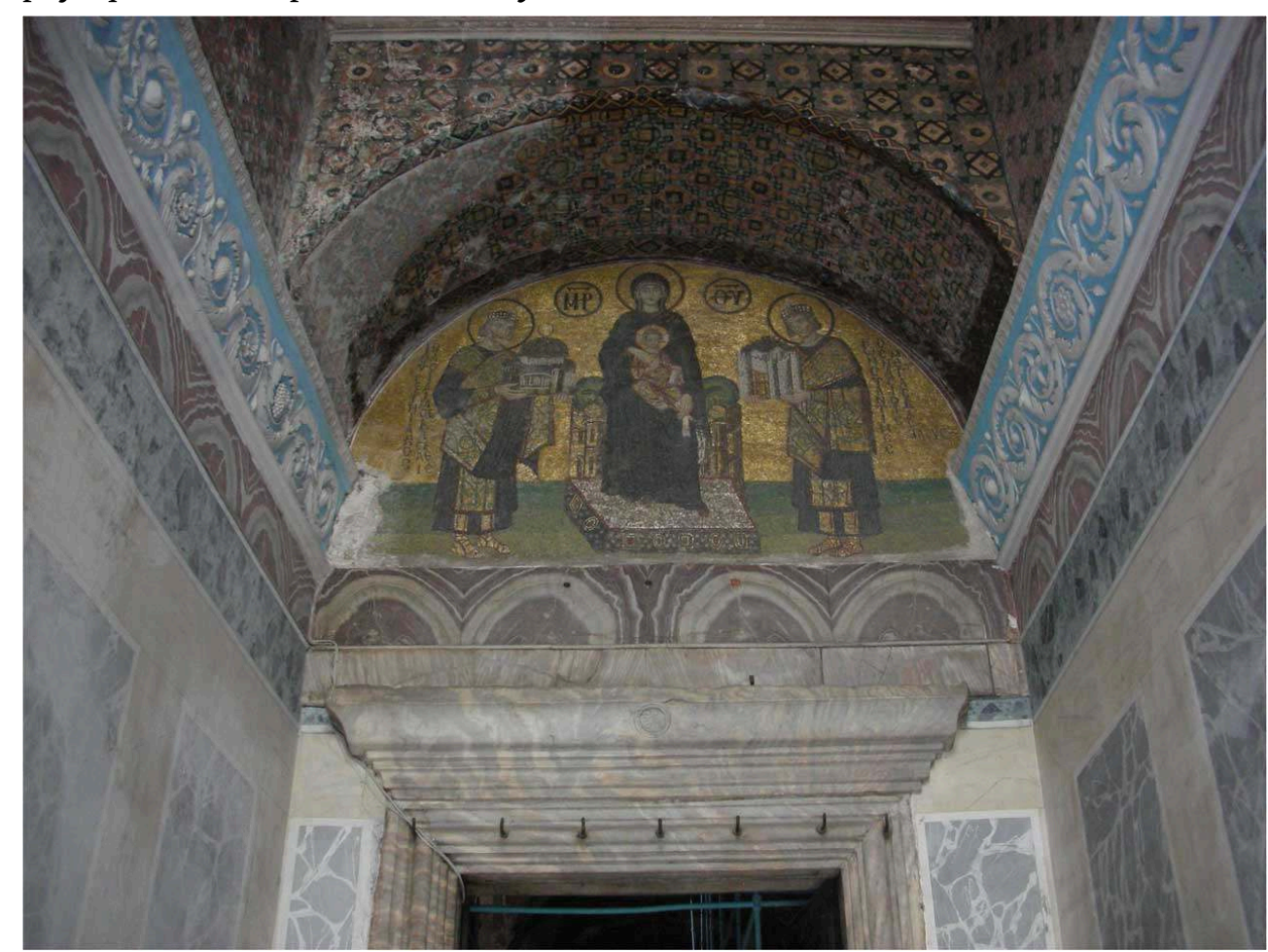

Victor Buchli

La mosaïque absidale de l'intronisation de Theotokos

Dans la basilique Sainte-Sophie, Istambul, 2009.

17 Comme nous l'avons dit plus haut, les idoles se caractérisent, quant à elles, par une conception visuelle et physique de la co-présence. C'est-à-dire qu'elles incarnent la divinité, et que cette dernière peut être endommagée si l'idole est détériorée ou détruite. Comme les archéologues et les anthropologues de la tradition euroaméricaine, d'aucuns ressentent la même chose lorsqu'un artefact est détruit. La destruction d'un héritage culturel commun est un acte de vandalisme scandaleux qui constitue une perte et un véritable dommage pour nos communautés. Cela s'explique par le fait que, dans les deux cadres, il existe une conception similaire du registre matériel et $d u$ sensorium en termes de présence plutôt que d'autres formes de propinquité qui sont atteintes en termes de co-présence visuelle et physique. Lorsqu'une idole est détruite, la divinité est affectée; lorsqu'un monument culturel est détruit, la culture est irrévocablement perdue. 


\section{Vision et matérialité}

La conception de la vision par rapport aux registres matériels spécifiques est particulièrement instructive dans les contextes chrétiens anciens, en particulier la tradition chrétienne qui s'est focalisée sur trois éléments matériels que les spécialistes de Byzance appellent «le pain, les os et les images », qui renvoient respectivement à l'Eucharistie, aux reliques et aux icônes (Barber 2002). Il est ici important de distinguer deux formes de regard : « regarder vers » opposé à « regarder au-delà » (Frank 2000) ou "être orienté vers» pour Barber 2002). Les prépositions sont ici cruciales pour comprendre les implications de ce registre matériel où l'on peut "regarder au-delà » ou « regarder à travers" plutôt que « regarder vers ». Dans cette perspective, l'œil possédait de tels pouvoirs de pénétration que le bois et la peinture de l'icône ne constituaient pas d'obstacle pour regarder au-delà de l'image et appréhender le prototype. Dans un tel cas, au sein de ce registre matériel, la surface des choses ne constitue pas un ensemble de signes derrière lesquels il y aurait un sens, c'est-à-dire un registre matériel derrière lequel repose un sens immatériel, mais plutôt une production de sens avec les relations nouvelles qui en découlent (voir également Pinney 2005; Henare \& al. 2007).

Cette forme de vision " unifiante » et tactile a pour fonction, dans le contexte de la dernière époque byzantine, d'unifier et de reconfigurer "le pain, les os et les images ", qui fournissent et facilitent le contact avec le divin, choses qui, selon les conceptions modernes, constitueraient des corpus distincts de culture matérielle. Le regard haptique lié au pain, aux os et aux images actualise le sacré absent. Il devient une technique locale extrêmement puissante pour "présentifier» l'absence. Dans ces conceptions chrétiennes anciennes, il est admis que « le pain, les os et les images » sont en eux-mêmes dénués d'importance. L'efficacité ne repose pas dans les objets euxmêmes mais dans le pouvoir haptique de l'œil du spectateur. Mais cet œil n'est pas passif. Il est capable de «toucher » le divin, d'entrer en contact physique avec lui (voir également Eck 1998, sur Darshan et la vision haptique).

Concernant «les os », les premiers Pères de l'Église, comme Saint Jérôme par exemple, ont reconnu qu'une relique n'est rien de plus que «de la poussière recouverte d'un linge précieux » (Saint Jérôme in Frank 2000 : 176). C'est néanmoins l'œil haptique du spectateur qui faisait des reliques l'incarnation d'un prophète: les peuples venaient " au-devant de ces reliques avec autant de joie que s'ils eussent vu un prophète vivant " (Saint Jérôme in Frank 2000: 176). La vision haptique représentait la réalité de la présence du prophète, en reconstituant visuellement une forme à partir d'éléments différents. De même, l'icône/image est selon Barber « un moyen d'étendre le toucher de la relique par le biais d'une réitération tangible " (Barber 2002 : 23). Une icône est à la fois une représentation et une relique, elle est à la fois un original et une copie - une copie dans le sens où elle est une reproduction d'une image prototypique originale et une relique dans le sens "haptique " où elle a eu un "contact» physique avec le prototype et possède par conséquent un aspect étendu et diffusé de l'original.

Cette notion de "regarder à travers" opposée au "regarder vers" suggère une relation tactile et active, désagrégeante, avec le monde matériel/visuel. Il s'agit d'une relation interpénétrante, dans laquelle il est difficile de discerner où une chose finit et où une autre commence. Ce ne sont pas des surfaces d'interrelation stables, mais des surfaces hautement poreuses à travers lesquelles il est possible de faire des offrandes et 
où des entremêlements hybrides peuvent être réalisés. Si on "regarde au-delà » de ce monde, ce n'est pas tellement pour parvenir sous la surface des choses, mais plutôt pour passer à travers elles et s'engager dans le «canal » de la divinité. C'est ce que les conceptions chrétiennes anciennes de la matérialité ont essayé de rendre plus accessible. Ainsi, la relique/image est utilisée au sein d'un registre particulier de proximités qui est facilité par la vision haptique. L'objectif est d'unifier et d'actualiser le divin par une relation analogique, soit à travers une partie du corps, soit à travers un artefact proche du corps en addition à une ressemblance mimétique formelle par rapport au prototype, comme c'est le cas par exemple dans les conventions formelles qui régissent la réalisation des icônes.

21 Le statut de l'Eucharistie et du "pain » est négocié d'une autre façon. Les controverses byzantines des $\mathrm{VIII}^{\mathrm{e}}$ et $\mathrm{IX}$ siècles à propos de l'iconoclasme n'étaient pas des débats sur la nature idolâtre des icônes et le péché d'idolâtrie mais soulevaient une question technique : comment mieux actualiser (ou plutôt établir le contact avec) le divin et ancrer les relations sociales dans le présent sur terre. La question n'était pas de savoir à quel point une icône pouvait être véritable, mais comment le monde matériel pouvait, de la meilleure manière possible, s'harmoniser avec l'infini (Barber 2002 : 59). En bref, quel type de proximité (avec ses registres sensoriels afférents) peut actualiser le divin le plus efficacement possible. Les icônes et les représentations des divinités n'étaient pas mauvaises en elles-mêmes, l'essentiel est de savoir si elles assuraient ou pas les fonctions qu'elles étaient censées remplir. Les iconophiles ont souligné la façon dont la relation haptique matérialise le divin, visuellement, autant que physiquement. Cette idée fut cependant remise en cause par les iconoclastes qui avaient avancé l'idée que l'efficacité du support matériel de l'icône (ou de la relique) était limitée. L'icône (ou la relique) n'était pas à la hauteur de la tâche consistant à présentifier le divin, puisqu'elle était d'origine non divine, matérielle et terrestre (bois, peinture, etc.). Or, le Conseil iconoclaste de 754 déclara que l'Eucharistie était le moyen le plus efficace d'actualiser le divin, car le Christ, lors du Dernier repas, avait dit à propos du pain et du vin : « ceci est mon corps » et « ceci est mon sang » (Barber 2002 : 79).

L'image peinte n'a jamais été en mesure d'établir cette connexion directe en termes de proximité relationnelle avec la divinité du Christ. La présence de ces paroles du Christ dans les Évangiles indique cependant une possibilité plus directe d'actualiser le divin à travers les actions mimétiques de l'Eucharistie. Puisque le Christ a consacré le pain et le vin comme des représentations de sa chair et de son sang, l'empereur Constantin $\mathrm{V}$ pouvait définir l'Eucharistie comme une icône et un moyen plus efficace de présentifier le divin: "Le pain que nous mangeons est aussi une icône de son corps, puisqu'il a façonné sa chair de sorte qu'elle est devenue une figuration de son corps » (Barber $2002: 80)$.

Si le Christ lui-même a considéré que ces formes matérielles constituaient des représentations appropriées, alors elles sont supérieures à toutes les autres formes matérielles (comme le bois et la peinture) qui ne furent pas consacrées par le Christ à l'origine (voir Ginzburg 2002 ; Engelke 2005 ; Vilaça 2005). Nous sommes ici dans une situation où la propinquité est atteinte par sa relation avec la mimesis et par l'analogie avec l'Eucharistie. 


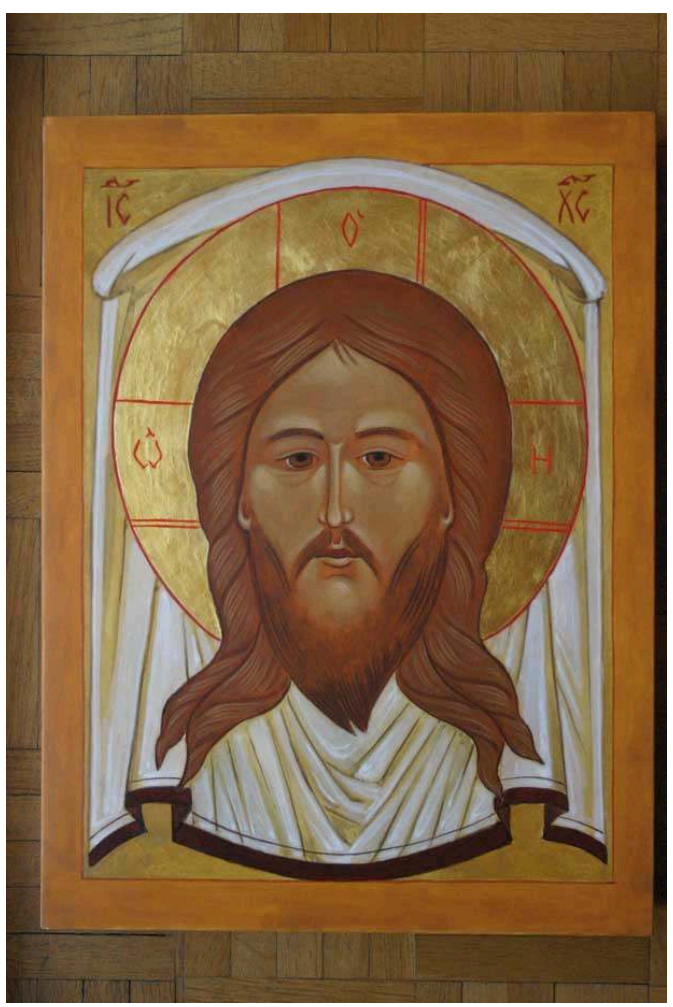

IICÔNE DE LA SAINTE FACE $30 \times 40 \mathrm{~cm}$. Réalisation Anne Philippenko.

(C) A. Philippenk

Des iconophiles tels que Nicéphore ont cependant plaidé pour l'efficacité des icônes figuratives en termes de proximité et d'analogie mimétique :

«L'icône est liée à l'archétype, elle entretient avec ce dernier une relation de cause à effet. Donc, l'icône est forcément une parente et peut être désignée comme telle. Une parente tire son nom du fait qu'elle est constituée d'autre chose, et que dans la relation, les deux sont liées par la réciprocité. La ressemblance est une relation intermédiaire qui fait le pont entre les extrêmes, à savoir les deux objets dont l'un ressemble à l'autre, les deux étant unifiés et connectés par la forme, bien qu'ils soient de nature différente » (Nicéphore in Barber $2002: 116$ ).

La visualité haptique est donc capable de lier ensemble des matériaux incompatibles, des matériaux bruts comme le bois avec le divin (la figure du Christ) par le biais de l'œuvre -mimétique de l'icône figurative et, plus important, de l'œil constitutif du spectateur. En outre, Nicéphore avance, en relation avec les mécanismes des icônes, qu'« en rendant l'absent présent par la manifestation de la similarité et du souvenir de la forme, [l'icône] entretient [avec son archétype] une relation ininterrompue durant toute son existence » (Nicéphore in Barber 2002 : 119), atteignant ainsi la propinquité et la présence par des moyens nouveaux.

Les deux technologies du prototype suggèrent ici des registres matériels très différents qui servent à actualiser de deux façons sensorielles distinctes. Ces technologies ne produisent pas une densité « morale » et « matérielle » unifiée (Durkheim [1912] 2008) comme nous l'interprétons traditionnellement dans les sciences sociales. Pourquoi un registre particulier fonctionnerait-il et pas un autre ? Il me semble que c'est le fait d'un " engagement" (au sens où le philosophe des sciences Joseph Rouse l'entend) allant dans le sens de l'efficacité du registre et de la communauté qui est produite par le biais de cet « engagement» (Rouse 2002). Pour ces raisons, je suis en faveur de l'idée que nos 
conceptions conventionnelles de la présence empirique gagneraient à être reconsidérées dans l'optique de la proximité et des formes de communautés qu'elle permet, plutôt qu'en termes de réalité empirique au sens courant, où la co-présence visuelle et physique peut être considérée comme une simple forme de proximité parmi de nombreuses autres.

\section{L'immatériel et le présent absent}

La question du présent absent semble tout aussi paradoxale que l'idée d'immatérialité mais les deux sont liées. Dire que quelque chose est là mais n'est pas présent revient simplement à privilégier un engagement sensoriel par rapport à un autre. En partant de ces prémisses, il me semble qu'il s'agit ici d'un engagement visuel de proximité physique - nous sommes tous co-présents, physiquement proches et visuellement disponibles à tous, en termes de proximité corporelle, lorsque nous nous trouvons dans la même pièce, le même bâtiment, le même village, etc., comme nous pouvons le concevoir dans les termes empiriques du sens commun, c'est-à-dire en termes de densités «morales » et «matérielles » qui constituent la vie sociale (Durkheim [1912] 2008).

Il vaut aussi la peine de noter qu'il est évident, sur le plan ethnographique, que ce qui est visuellement co-présent, ce que nous pourrions autrement appeler la "réalité empirique » dans la tradition euroaméricaine courante, est souvent interprété comme étant résolument irréel ou au moins extrêmement instable. D'aucuns pensent que ce qui est réel, stable et durable va au-delà de ce qui est physiquement co-présent : le royaume des ancêtres, les connexions totémiques, les cosmologies constitutives, la présence de Dieu, etc. Le corps et le monde matériel émergent et se décomposent - ils sont instables -; ce qui est néanmoins stable, ce sont les principes cosmologiques qui structurent le monde. Dire que quelque chose est présent tout en étant absent revient à concevoir cette absence en termes de co-présence visuelle et physique. Les ancêtres, les morts, la cosmologie ou Dieu sont tout à fait présents, mais ils ne sont pas appréhendés dans les termes de co-présence physique et visuelle que désigne la "présence absente ». Par conséquent, diverses technologies complexes ont été élaborées (communément nommées "activités rituelles») pour actualiser ces entités au sein de ce registre matériel donné qui, dans un autre contexte, par exemple celui de l'empirique commun, pourrait être considéré comme absent. La présence peut être rendue dans beaucoup de registres matériels au-delà du co-présent visuel et physique. Elle peut fonctionner selon un grand nombre de conceptions différentes des sens et des hiérarchies de savoir que nous associons à des sens différents, pour des époques historiques et des contextes sociaux différents, ainsi que Claassen et Howes l'ont montré dans leurs travaux sur l'anthropologie des sens (Claassen 1993; Claassen \& Howes 2006 ; Howes 2005).

\section{Prototype et propinquité}

Les deux formes du prototype évoquées dans cet article sont impliquées dans deux formes d'universalisme transcendant: le prototype chrétien essaye d'actualiser et de soutenir une universalité et une entité : l'écoumène chrétien, sur des échelles de temps, de lieu et de tradition locale qui transcendent ceux de la co-présence visuelle et 
physique immédiate. Les exemples de Prototypage rapide ou d'impression en trois dimensions du début du xxI siècle suggèrent des processus analogues. Les deux technologies, ancienne et moderne, représentent une matérialité universalisante et non délimitée, d'une portée et d'une puissance extraordinaires, intimement liées aux économies politiques de leur époque. Les deux technologies exploitent également ce que nous appelons, avec une certaine ambivalence, l'« immatériel » afin de mettre en œuvre leur pouvoir et le contrôle qu'elles exercent respectivement sur le monde matériel. En outre, ces deux technologies créent des cadres contenant des dualismes productifs, qui reconfigurent radicalement nos relations, tant au niveau physique que politique, économique et sensoriel avec le monde qui nous entoure. D'une manière générale, la dichotomie présence/absence comprend une certaine acception du registre matériel et de la hiérarchie sensorielle, qui désigne en fait le présent visuel, corporel et physique. Notre conception de la réalité empirique se fait généralement selon ces termes. On pourrait voir cela comme un simple aspect de la propinquité - comme un moyen par lequel le voisinage et la proximité sont atteints en plus de l'analogie, de la relation et de la proximité temporelle et spatiale. Ce que nous considérons normalement comme de l'«immatériel " peut être envisagé simplement comme une conséquence importante d'un dualisme socialement productif.

\section{Parenté et présence}

Les deux exemples du prototype présentés dans cet article montrent -comment notre conception de la présence en relation avec le matériel gagnerait à être abordée en termes de propinquité. Nos conceptions conventionnelles de la présence, qui privilégient la co-présence visuelle et physique dans la tradition euroaméricaine, sont troublées par le fonctionnement du prototype dans le contexte du début du christianisme ancien et de l'ère moderne récente. Cependant, lorsqu'on se penche sur ces deux technologies différentes du prototype, il faut prendre en compte les différents registres matériels et sensoriels qui sont en jeu. D'un côté, la présence peut être facilitée dans des registres matériels radicalement différents qui atteignent la propinquité, tels que la technologie des icônes durant la période chrétienne ancienne. Ici, les concepts païens de temps et d'espace sont reconfigurés par les technologies universalisantes et radicalement restructurantes du prototype chrétien pour créer des formes nouvelles de communautés, selon les termes de l'écoumène chrétien universel, et avec lui des conceptions nouvelles de l'espace et du temps. D'un autre côté, les formes nouvelles de proximité liées aux nouvelles technologies contemporaines comme la Fabrication rapide (qui remet en cause les conceptions conventionnelles de la coprésence visuelle et physique) contribuent à troubler davantage les catégories euroaméricaines de temps, d'espace et de matérialité, notamment en raison du caractère radicalement "perturbateur" de ces technologies. Les dualismes qui insistent sur la séparation radicale du matériel vis-à-vis de l'immatériel et les normes « incorrigibles » de l'immatériel (Rorty 1970 ; Thalberg 1983) sont ici questionnés eux aussi par les nouveaux « engagements » émergents et les entremêlements sociaux qui commencent tout juste à devenir des sujets d'études. Les catégories analytiques, telle que la «culture matérielle» dont le statut constitue une catégorie empirique et ontologique soutenue par ces objectivations, sont abordées d'une façon nouvelle. Comme on peut le constater avec l'indistinction matérielle et discursive du code binaire « immatériel » et de la « chose matérielle » indépendante, telle qu'elle est représentée 
par la Fabrication rapide et les extraordinaires capacités productives des "poudres sans valeur " (Barad 1998), une nouvelle conception du matériel et de l'immatériel émerge. Nos conceptions conventionnelles de la présence et de l'absence ne permettent de les comprendre que comme un aspect particulier et socialement contingent et productif de propinquité parmi de nombreux autres.

\section{Detail .MGX}

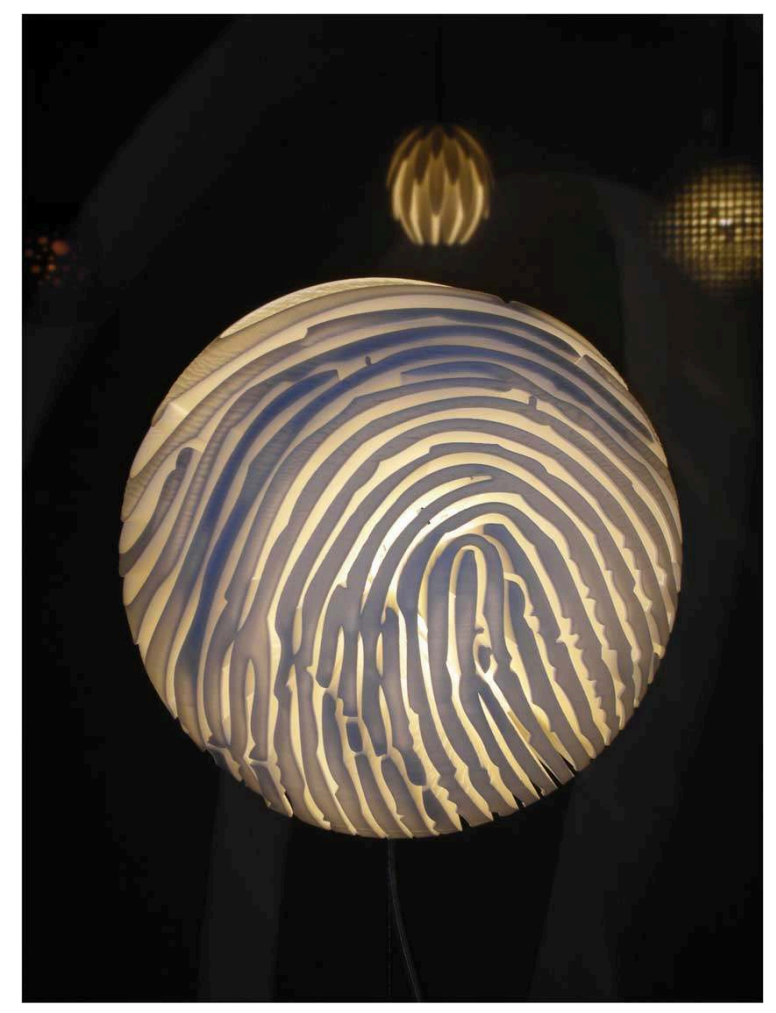

(B) Materialise

\section{BIBLIOGRAPHIE}

Lustre créée par Luc Merx, fabriqué par.MGX pour Materialise.

Barad, Karen, 1998, « Getting Real : Technoscientific Practices and the Materialization of Reality », Differences 10 (2) : 87-128.

Barber, Charles, 2002, Figure and Likeness : On the Limits of Representation in Byzantine Iconoclasm. Princeton, Princeton University Press.

Bille, Mikkel, Hastrup, Frida \& Sørensen, Tim Flohr eds., 2010, An Anthropology of Absence. Materializations of Transcendence and Loss. New York, Springer Press.

Claassen, Constance, 1993, Worlds of Sense : Exploring the Senses in History and Across Cultures. Londres, Routledge. 
Claassen, Constance \& Howes, David, 2006, « The Museum as Sensescape : Western Sensibilities and Indigenous Artifacts », in E. Edwards, C. Gosden \& R. Phillips eds., Sensible Objects : Colonialism, Museums and Material Culture. Oxford, Berg.

Durkheim, Emile, 2008 [1912], Les Formes élémentaires de la vie religieuse. Paris, PUF.

Eck, Diana L., 1998, Darçan : Seeing the Divine Image in India. Columbia University Press.

Engelke, Matthew, 2005, « Sticky Subjects and Sticky Objects » in D. Miller ed., Materiality. Durham, Duke University Press.

Frank, Georgia, 2000, The Memory of the Eyes : Pilgrims to Living Saints in Christian Late Antiquity. Berkeley, University of California Press.

Gell, Alfred, 2009 [1998], L'Art et ses agents. Dijon, Les Presses du réel.

Ginzburg, Carlo, 2002, Wooden Eyes : Nine Reflections on Distance. Londres, Verso.

Henare, Amira, Holbraad, Martin \& Wastell, Sari eds., 2007, Thinking Through Things : Theorising Artefacts Ethnographically. Londres, Routledge.

Hopkinson, Neil \& al. eds, 2006, Rapid Manufacturing and Industrial Revolution for the Digital Age. Chichester, John Wiley \& Sons Ltd.

Howes, David ed., 2005, Empire of the Senses. Oxford, Berg.

Keane, Webb, 2005, «Signs are Not the Garb of Meaning : On the Social Analysis of Material Things », in D. Miller ed., Materiality. Durham, Duke University Press : 182-205.

Miller, Danny, 2005, « Materiality : an Introduction », in D. Miller ed., Materiality. Durham, Duke University Press.

Ong, Walter, 1967, The Presence of the Word: Some Prologemena for Cultural and Religious History. New Haven, Yale University Press.

Pinney, Christopher, 2005, « Visual Culture » in V. Buchli ed., The Material Culture Reader. Oxford, Berg Publishers.

Povinelli, Elisabeth, 2001, « Radical Worlds : The Anthropology of Incommensurability and Inconceivability », Annual Review of Anthropology 30 : 319-34.

Rorty, Richard, 1970, « Incorrigibility as the Mark of the Mental », Journal of Philosophy, LWVII : 399-424.

Rose, Nikolas, 1998, Inventing our Selves : Psychology, Power and Personhood. Cambridge, Cambridge University Press.

Rouse, Joseph, 2002, How Scientific Practices Matter : Reclaiming Philosophical Naturalism. Chicago, University of Chicago Press.

Rowlands, Michael, 2005, « A Materialist Approach to Materiality », in D. Miller ed., Materiality. Durham, N.C., Duke University Press.

Sassen, Saskia, 2007, Territory, Authority, Rights : From Medieval to Global Assemblages. Princeton, Princeton University Press.

Thalberg, Irving, 1983, Immateriality, Mind, vol. XCII : 105-13. 


\section{NOTES}

1. Cet article est dédié à la famille Mathé, de Paris, pour sa grande gentillesse. Il est en partie issu du chapitre intitulé «Presencing the Immaterial » in Bille, Mikkel, Frida Hastrup \& Tim Flohr Sørensen, eds., 2010, An Anthropology of Absence. Materializations of Transcendence and Loss. New York, Springer Press.

\section{RÉSUMÉS}

La culture matérielle, la numérisation et le problème de l'artefact. Les récentes innovations dans le domaine de la numérisation de la fabrication d'objets posent de nouvelles questions concernant notre conception de la nature de l'artefact. Elles posent également la question de l'élargissement de notre conception de la culture matérielle. Cet article a pour objet d'examiner l'émergence de la Fabrication rapide dans le domaine du Prototypage rapide, ce qui permettra d'appréhender la question de la numérisation et, plus généralement, notre conception de la culture matérielle. Étant donné qu'il s'agit d'une technologie fondée sur le concept de prototype, je la mettrai en parallèle avec une conception plus ancienne de prototype, à savoir le prototype chrétien, dans le but d'aborder les questions plus larges de matérialité et de présentification dans le cadre de ces deux technologies qui sont apparues au début et à la fin de l'ère chrétienne. Mon propos se focalisera plus particulièrement sur la question de l'immatériel, qui est au cœur des deux technologies du prototype, et sur la façon dont une technologie particulière de présentification liée à certaines conceptions de l'immatérialité exemplifie les dualismes productifs nécessaires à la structure de la vie sociale (Miller 2005).

Material Culture, Digitization and the Problem of the Artefact. Recent innovations in the digitization of manufacturing have posed new problems for how we understand the nature of the artefact and the way material culture might be conceived more broadly. In this paper I want to address the emergence of Rapid Manufacturing from the area of Rapid Prototyping to consider the question of digitisation and our understandings of material culture more broadly. As this is a technology based on the concept of the prototype I want to consider it alongside a more ancient technology of the prototype, that is the Christian Prototype to engage larger questions of materiality and presencing that the two technologies address and which have emerged at the beginning and end of our Common Era. In particular I want to focus on the question of the immaterial which is at the heart of both technologies of the prototype and how a particular technology of presencing dependant on contingent understandings of the immaterial construct the productive dualisms necessary for the structure of social life (Miller 2005).

\section{INDEX}

Mots-clés : culture matérielle, fabrication rapide, icône, immatériel, présence, propinquité

Keywords : icons, immaterial, material culture, presence, propinquity, rapid manufacturing 


\section{AUTEURS}

\section{VICTOR BUCHLI}

University College London

v.buchli@ucl.ac.uk 